\title{
AVALIAÇÃO QUALI-QUANTITATIVA DA ARBORIZAÇÃO NA PRAÇA AGOSTINHO NOHAMA, BAIRRO LAUZANE PAULISTA, SÃO PAULO - SP
}

Rita de Cássia Sousa ${ }^{1}$; Osny Tadeu de Aguiar ${ }^{2}$; Lucas Teixeira Alves da Silva ${ }^{3}$; Laís Alves Silva ${ }^{3}$ : Raquel

$$
\text { Cristina } \text { Marra }^{3} 4
$$

\section{RESUMO}

A vegetação urbana influencia as condições climáticas e biológicas de determinado local, e podem trazer inúmeras contribuições ecológicas, econômicas e sociais. Portanto, objetivou-se avaliar quali-quantitativamente a arborização da Praça Agostinho Nohama, localizada na zona norte do município de São Paulo (SP), para garantir os benefícios proporcionados pelas áreas verdes. Para tanto, foram inventariados todos os indivíduos arbustivos e arbóreos com circunferência a altura do peito (CAP) $\geq 10 \mathrm{~cm}$, e observaram-se características físicas, biológicas e fitossanitárias. Foram quantificados 333 indivíduos vivos, distribuídos em 85 espécies, pertencentes a 67 gêneros de 36 famílias. Há cultivo de espécies exóticas no local. Sobre as condições gerais dos espécimes amostrados, 289 indivíduos $(85,3 \%)$ estão em bom estado de conservação, 39 (11,5\%) necessitam de cuidados por apresentarem injúrias físicas e/ou biológicas e, $11(3,2 \%)$ precisam ser removidos, por oferecerem risco aos seus frequentadores. A Praça apresenta importante papel ecológico, por interagir com líquens, insetos, epífitas e aves. A fim de contribuir com a diversidade regional e possibilitar atividades de educação ambiental, sugere-se um plantio das espécies nativas do complexo, Cantareira.

Palavras-chave: Arborização urbana; Fitossanidade; Paisagismo.

\section{QUALI-QUANTITATIVE VALUATION OF THE SQUARE ARBORIZATION AGOSTINHO NOHAMA, DISTRICT LAUZANE PAULISTA, SÃO PAULO - SP}

\section{ABSTRACT}

The urban vegetation influences climate and biological conditions of a particular site, and can provide numerous ecological, economic and social contributions. The objective of the study was to evaluate quantitatively and qualitatively the afforestation of the Agostinho Nohama Square, located in the northern city of São Paulo (SP), to ensure the benefits provided by green areas. For this, individuals of all shrubs and tress with cincumference at breast height $(\mathrm{CAP}) \geq 10 \mathrm{~cm}$ were inventoried, and the physical, biological and phytosanitary features were observed. We quantified 333 living individuals, distributed in 85 species belonging to 67 genres of 36 families. There is cultivation of exotic species on the site. About the general condition of the specimes sampled, 289 individuals $(85,3 \%)$ are in good condition, $39(11,5 \%)$ require special attention due to physical and/or biological injuries, and $11(3,2 \%)$ need to be removed by offering risk to the visitors of the square. The Square provides an important ecological role by interacting with lichens, insects, epiphytes and birds. In order to contribute to regional diversity and enable environmental education activities, it is suggested a planting of native species of the Cantareira region.

Key words: Urban forest; Phytosanitary; Garden design.

\footnotetext{
${ }^{1}$ Bióloga e Especialista em Engenharia Ambiental. Rua Des. Rodrigues Sette, 365, apto 84, bloco 9, CEP: 02634-070. rcsousa83@gmail.com

${ }^{2}$ Biólogo e Mestre em Recursos Florestais, Pesquisador do Instituto Florestal do Estado de São Paulo, Divisão de Dasonomia, Seção de Madeira e Produtos Florestais. Rua do Horto, 931. Cx.p.:1322 CEP: 02377-000. tadeu@if.sp.gov.br

${ }^{3}$ Graduandos em Ciências Biológicas, Universidade São Judas Tadeu, Faculdade de Ciências Biológicas e da Saúde.

${ }^{4}$ recebido em 24.05.2012 e aceito para publicação em 15.03.2014
} 
O desenvolvimento urbano altera significativamente o ambiente. No final do século XIX o município de São Paulo foi modificado, as várzeas, campos e florestas foram substituídos pela grande metrópole. O crescimento populacional desordenado traz diversos problemas ambientais, como: poluição atmosférica, hídrica, sonora e visual, redução da cobertura florestal nativa, além da impermeabilização do solo (USTERI, 1991 apud TAKIYA, 2002; ROCHA et al., 2004; FILHO e RIBEIRO, 2006; SEADE, 2010).

O Atlas Ambiental do Município de São Paulo mostra que $48 \%$ da Região Metropolitana de São Paulo (RMSP) apresentam carência significativa de cobertura vegetal, provavelmente a realidade é outra após nove anos de crescimento. Estudos apontam fragilidade na fiscalização, onde a urbanização paulista é liderada por loteamentos clandestinos que não seguem a lei vigente, isto é, na maioria dos lotes com áreas verdes, ocorre supressão arbórea (TAKIYA, 2002).

A vegetação em área urbana disposta em bosques, praças, entre outros, representa um aspecto positivo no âmbito psicológico coletivo, por ter relação com a prática de atividades recreativas e pela proximidade, com a natureza (SANCHOTENE, 1994).

A arborização urbana influencia as condições climáticas e biológicas de determinado local (ABREU, 2008). Sua importância está ligada às funções estéticas, ecológicas, econômicas e sociais (PAIVA, 2006) como exemplo, espécies arbóreas podem ser utilizadas como biomonitores de baixo custo, por reter e absorver poluentes atmosféricos (MOREIRA, 2010). Auxiliam na captura do gás carbono, principalmente os exemplares nativos de crescimento moderado e rápido, pela capacidade de absorverem mais esse gás, transformando-o em biomassa vegetal (MUNEROLI e MASCARÓ, 2010), As copas interceptam parte da precipitação que auxilia o controle de enchentes (SILVA, 2008). Árvores influenciam a temperatura e a umidade relativa do ar, atuando numa escala microclimática, onde, pode proporcionar sensação de conforto térmico (ABREU e LABAKI, 2010).

O inventário arbóreo é fundamental para garantir os benefícios proporcionados pelas áreas verdes (TAKAHASI, 1994). Por meio dos levantamentos quantitativos, pode-se se conhecer, além da riqueza, a frequência dos indivíduos e a quantidade total de árvores (SOUZA e BUENO, 2000), possibilitando um manejo adequado e possibilidade de monitoramento. Santamour Junior (1990) afirma que para garantir o máximo de proteção contra pragas e doenças é essencial a maior diversidade de espécies de árvores na paisagem urbana, não ultrapassando mais que $10 \%$ da mesma espécie, $20 \%$ de um gênero e $30 \%$ de uma família botânica. Dados quantitativos isolados expressam parte dos dados sobre a vegetação urbana, e para completar as informações são necessárias análises combinadas (quali-quantitativas) possibilitando considerações úteis, baseado em resultados que caracterizam a distribuição e qualidade sanitária dos indivíduos arbóreos (MILANO et al., 1992).

Para a prevenção de problemas fitossanitários, o manejo adequado das árvores é fundamental. Estudos mostram que podas executadas inadequadamente são causas de ataque de fungos, cupins xilófagos e brocas-de-madeira em árvores de pau-brasil e sibipiruna (AMARAL et al., 2003).

Mediante as inúmeras contribuições que as áreas verdes proporcionam ao meio urbano, objetivou-se avaliar quali-quantitativamente a arborização da 
Praça Agostinho Nohama (São Paulo, SP), a fim de fornecer resultados relevantes sobre a qualidade ambiental da Praça, e se necessário, propor intervenções para sua melhoria.

\section{MATERIAL E MÉTODOS}

Este estudo foi realizado na Praça Agostinho Nohama (23W 28' 07" e 46S 38' 28"), localizada no bairro Lauzane Paulista, zona norte de São Paulo e possui uma extensão de 458,32 metros.

O clima da região é classificado como Tropical Úmido Serrano da Cantareira, onde as altitudes variam de 800 a 1200 metros, que proporciona uma condição térmica amena, com temperaturas médias anuais de $17,7^{\circ}$ a $19,3^{\circ} \mathrm{C}$, e precipitações médias anuais entre 1400 a $1590 \mathrm{~mm}$ (TARIFA e ARMANI, 2000).

\section{Inventário Quantitativo}

Os trabalhos de campo foram realizados no mês de setembro de 2011, e todos os indivíduos arbustivos e arbóreos com circunferência a altura do peito (CAP) maior e igual a $10 \mathrm{~cm}$ foram amostrados. Os espécimes foram plaqueados com numeração crescente, para diminuir a probabilidade de erros

\section{Avaliações Qualitativas}

Para todos os indivíduos quantificados foram observadas características físicas e biológicas de acordo com as seguintes definições:

Equilíbrio geral: Para esta característica observaram-se o fuste e a copa, podendo ser; equilibrado, aquele que possui eixo simétrico ou desequilibrado, aquele que apresenta eixo assimétrico.
A Praça situa-se a poucos metros do Parque Estadual Alberto Löfgren (Horto Florestal) que por sua vez, está interligado ao Parque Estadual da Cantareira, formando um maciço de área verde na zona norte de São Paulo.

Para a realização do inventário quali-quantitativo da praça, foi utilizada a metodologia proposta por Silva Filho (2002) apud Bortoleto (2004), com adaptações.

amostrais. As identificações (nome científico e popular) foram feitas com o auxílio de taxonomistas, além de consultas às bibliografias específicas. Apenas uma pessoa realizou a estimativa da variável altura, e o CAP foi medido com auxílio de uma fita métrica.

Aspectos fitossanitários: Analisaram-se a olho nu a presença de "pragas" como insetos (formiga, lagarta, pulgão, broca, cupim, entre outros), fungos e doenças.

Local de ataque: Verificaram-se qual a parte do espécime vegetal foi afetado por insetos, fungos e/ou doenças; Caule, Raiz, Ramos, Folhas, Frutos e/ou Flores. 
Injúrias: Quando observada qualquer lesão provocada, seja pelos agentes patogênicos ou mecânicos, esta foi classificada em Grave (compromete a sobrevivência do indivíduo), Média (o indivíduo pode ser recuperado mediante ações) ou Leve (pequena proporção), verificaram-se também injúrias por Vandalismo (inscrições e/ou assolações, ocasionadas por seres humanos).

Ecologia: Observaram-se as presenças de insetos, liquens, epífitas, e pássaros.

Fenologia: Anotaram-se características que inferem sazonalidade dos indivíduos amostrados, isto é, a parte vegetal presente na fase do levantamento; folhas, flores, e/ou frutos.

Estado geral: a partir das características observadas anteriormente, os espécimes foram classificados pelas condições gerais, descritas a seguir.

\section{Análise dos dados}

A nomenclatura científica seguiu as normas propostas pelo APG II (Angiosperm Phylogeny Group II), segundo Souza e Lorenzi (2007). A grafia, sinonimização, origem, e distribuição fitogeográfica das espécies foram verificadas utilizando os bancos de dados Lista de espécies da Flora do Brasil (FORZZA et al., 2010) e W3 Tropicos (MOBOT, 2008).

Sobre a origem adotou-se a definição proposta por Ziller (2001), sendo espécies exóticas, aquelas de ocorrência fora dos limites geográficos historicamente conhecidos, neste caso, foram classificadas como exóticas aquelas que ocorrem naturalmente fora do estado de São Paulo e do Brasil.
Boa, indivíduo plenamente vigoroso e sadio, podendo apresentar algum sinal de deficiência superficial, porém em bom estado.

Regular indivíduo em declínio, com injúrias médias descaracterizando sua arquitetura, desequilibrando o vegetal.

Ruim, indivíduo em estado avançado e irreversível de declínio, com injúrias graves, com risco de queda ou morte do espécime.

Morta, indivíduo morto com risco de queda, perdendo sua função na arborização, além de ser um risco aos visitantes.

Os dados foram tabulados no programa Microsoft Excel ${ }^{\circledR}$. 


\section{Quantitativos}

Foram quantificados 333 indivíduos vivos e seis indivíduos mortos, totalizando 339 exemplares arbustivos e arbóreos, distribuídos em 85 espécies, pertencentes a 67 gêneros de 36 famílias, conforme Tabela 1.

Tabela 1. Espécies quantificadas na Praça Agostinho Nohama. Origem, Q (Frequência Absoluta), F\% (Frequência Relativa)

\begin{tabular}{|c|c|c|c|c|c|}
\hline Família & Espécie & Nome popular & Origem & $\mathbf{Q}$ & F\% \\
\hline Anacardiaceae & Mangifera indica L. & mangueira & nativa & 4 & 1,18 \\
\hline Anacardiaceae & Schinus molle L. & aroeira-salsa & nativa & 1 & 0,29 \\
\hline Anacardiaceae & $\begin{array}{l}\text { Schinus terebinthifolius } \\
\text { Raddi }\end{array}$ & aroeira-vermelha & nativa & 2 & 0,59 \\
\hline Apocynaceae & Nerium oleander L. & oleandro & exótica & 1 & 0,29 \\
\hline Araliaceae & $\begin{array}{l}\text { Dendropanax cuneatus } \\
\text { (DC.) Decne. \& Planch. }\end{array}$ & pau-de-tamanco & nativa & 7 & 2,06 \\
\hline Araliaceae & $\begin{array}{l}\text { Schefflera arboricola } \\
\text { (Hayata) Merr. }\end{array}$ & $\begin{array}{l}\text { cheflera- } \\
\text { manchada }\end{array}$ & exótica & 1 & 0,29 \\
\hline Araliaceae & $\begin{array}{l}\text { Schefflera heterophylla } \\
\text { (Wall. ex G. Don) } \\
\text { Harms }\end{array}$ & cheflera & exótica & 3 & 0,88 \\
\hline Araucariaceae & $\begin{array}{l}\text { Araucaria angustifolia } \\
\text { (Bertol.) Kuntze }\end{array}$ & $\begin{array}{l}\text { pinheiro-do- } \\
\text { paraná }\end{array}$ & nativa & 2 & 0,59 \\
\hline Arecaceae & $\begin{array}{l}\text { Archontophoenix } \\
\text { cunninghamii H. Wendl. } \\
\text { \& Drude }\end{array}$ & $\begin{array}{l}\text { palmeira- } \\
\text { seafórtia }\end{array}$ & exótica & 6 & 1,77 \\
\hline Arecaceae & Caryota urens L. & $\begin{array}{l}\text { palmeira-rabo- } \\
\text { de-peixe }\end{array}$ & exótica & 2 & 0,59 \\
\hline Arecaceae & $\begin{array}{l}\text { Dypsis lutescens (H. } \\
\text { Wendl.) Beentje \& J. } \\
\text { Dransf. }\end{array}$ & areca-bambu & exótica & 1 & 0,29 \\
\hline Arecaceae & $\begin{array}{l}\text { Livistona chinensis } \\
\text { (Jacq.) R. Br. ex Mart. }\end{array}$ & palmeira-leque & exótica & 1 & 0,29 \\
\hline Arecaceae & $\begin{array}{l}\text { Phoenix roebelenii } \\
\text { O'Brien }\end{array}$ & $\begin{array}{l}\text { tamareira-de- } \\
\text { jardim }\end{array}$ & exótica & 10 & 2,95 \\
\hline Arecaceae & $\begin{array}{l}\text { Syagrus romanzoffiana } \\
\text { (Cham.) Glassman }\end{array}$ & jerivá & nativa & 23 & 6,78 \\
\hline Asparagaceae & $\begin{array}{l}\text { Dracaena marginata } \\
\text { Hort. }\end{array}$ & $\begin{array}{l}\text { dracena-de- } \\
\text { madagascar }\end{array}$ & exótica & 1 & 0,29 \\
\hline Asteraceae & $\begin{array}{l}\text { Vernonanthura } \\
\text { divaricata } \\
\text { (Spreng.) H.Rob. }\end{array}$ & cambará & nativa & 2 & 0,59 \\
\hline Bignoniaceae & $\begin{array}{l}\text { Handroanthus } \\
\text { chrysotrichus (Mart. ex } \\
\text { DC.) Mattos }\end{array}$ & $\begin{array}{l}\text { ipê-amarelo-do- } \\
\text { cerrado }\end{array}$ & nativa & 1 & 0,29 \\
\hline Bignoniaceae & Handroanthus sp. 1 & ipê-amarelo & - & 4 & 1,18 \\
\hline Bignoniaceae & Handroanthus sp. 2 & & - & 1 & 0,29 \\
\hline Bignoniaceae & Handroanthus sp. 3 & & - & 3 & 0,88 \\
\hline Bignoniaceae & $\begin{array}{l}\text { Jacaranda mimosifolia } \\
\text { D. Don }\end{array}$ & $\begin{array}{l}\text { jacarandá- } \\
\text { mimoso }\end{array}$ & exótica & 1 & 0,29 \\
\hline
\end{tabular}




\begin{tabular}{|c|c|c|c|c|c|}
\hline Família & Espécie & Nome popular & Origem & $\mathbf{Q}$ & $\mathbf{F \%}$ \\
\hline Bignoniaceae & $\begin{array}{l}\text { Spathodea nilotica } \\
\text { Seem. }\end{array}$ & bisnagueira & exótica & 19 & 5,60 \\
\hline Bixaceae & Bixa orellana L. & urucum & nativa & 1 & 0,29 \\
\hline Casuarinaceae & Casuarina sp. & & exótica & 6 & 1,77 \\
\hline Combretaceae & Terminalia catappa L. & chapéu-de-sol & subespontânea & 15 & 4,42 \\
\hline Cupressaceae & Chamaecyparis sp. & & - & 1 & 0,29 \\
\hline Cupressaceae & $\begin{array}{l}\text { Cupressus sempervirens } \\
\text { L. }\end{array}$ & cipreste-italiano & exótica & 16 & 4,72 \\
\hline Cupressaceae & Cupressus sp. & & exótica & 1 & 0,29 \\
\hline Euphorbiaceae & $\begin{array}{l}\text { Alchornea sidifolia } \\
\text { Müll.Arg. }\end{array}$ & tapiá & nativa & 4 & 1,18 \\
\hline Fabaceae & $\begin{array}{l}\text { Caesalpinia echinata } \\
\text { Lam. }\end{array}$ & pau-brasil & $\begin{array}{l}\text { nativa (BR), } \\
\text { exótica (SP) }\end{array}$ & 3 & 0,88 \\
\hline Fabaceae & $\begin{array}{l}\text { Delonix regia (Bojer ex } \\
\text { Hook.) Raf. }\end{array}$ & flamboyant & exótica & 4 & 1,18 \\
\hline Fabaceae & $\begin{array}{l}\text { Enterolobium } \\
\text { contortisiliquum (Vell.) } \\
\text { Morong }\end{array}$ & tamboril & nativa & 5 & 1,47 \\
\hline Fabaceae & $\begin{array}{l}\text { Erythrina speciosa } \\
\text { Andrews }\end{array}$ & $\begin{array}{l}\text { mulungu-do- } \\
\text { litoral }\end{array}$ & nativa & 1 & 0,29 \\
\hline Fabaceae & Indeterminada 1 & & - & 1 & 0,29 \\
\hline Fabaceae & Indeterminada 2 & & - & 1 & 0,29 \\
\hline Fabaceae & Indeterminada 3 & & - & 1 & 0,29 \\
\hline Fabaceae & $\begin{array}{l}\text { Libidibia ferrea (Mart. } \\
\text { ex Tul.) L.P.Queiroz }\end{array}$ & pau-ferro & $\begin{array}{l}\text { nativa (BR), } \\
\text { exótica (SP) }\end{array}$ & 4 & 1,18 \\
\hline Fabaceae & $\begin{array}{l}\text { Machaerium nyctitans } \\
\text { (Vell.) Benth. }\end{array}$ & bico-de-pato & nativa & 2 & 0,59 \\
\hline Fabaceae & $\begin{array}{l}\text { Piptadenia gonoacantha } \\
\text { (Mart.) J.F.Macbr. }\end{array}$ & pau-jacaré & nativa & 5 & 1,47 \\
\hline Fabaceae & $\begin{array}{l}\text { Poincianella pluviosa } \\
\text { (DC.) L.P.Queiroz }\end{array}$ & sibipiruna & $\begin{array}{l}\text { nativa (BR), } \\
\text { exótica (SP) }\end{array}$ & 2 & 0,59 \\
\hline Fabaceae & Samanea sp. & & - & 1 & 0,29 \\
\hline Fabaceae & $\begin{array}{l}\text { Schizolobium parahyba } \\
\text { (Vell.) Blake }\end{array}$ & guapuruvu & nativa & 1 & 0,29 \\
\hline Fagaceae & Quercus robur L. & carvalho-inglês & exótica & 2 & 0,59 \\
\hline Indeterminada 1 & Indeterminada 1 & & - & 1 & 0,29 \\
\hline Indeterminada 2 & Indeterminada 2 & & - & 1 & 0,29 \\
\hline Lauraceae & Ocotea pichurim Kunth & canela & exótica & 1 & 0,29 \\
\hline Lauraceae & Persea americana Mill. & abacateiro & subespontânea & 5 & 1,47 \\
\hline Lythraceae & $\begin{array}{l}\text { Lafoensia pacari A.St.- } \\
\text { Hil. }\end{array}$ & dedaleiro & nativa & 2 & 0,59 \\
\hline Malvaceae & $\begin{array}{l}\text { Ceiba speciosa (A.St.- } \\
\text { Hil.) Ravenna }\end{array}$ & paineira & nativa & 7 & 2,06 \\
\hline Malvaceae & $\begin{array}{l}\text { Malvaviscus arboreus } \\
\text { Cav. }\end{array}$ & malvavisco & exótica & 2 & 0,59 \\
\hline Malvaceae & Pseudobombax sp. & & - & 4 & 1,18 \\
\hline Melastomataceae & $\begin{array}{l}\text { Tibouchina granulosa } \\
\text { (Desr.) Cogn. }\end{array}$ & quaresmeira & $\begin{array}{l}\text { nativa (BR), } \\
\text { exótica (SP) }\end{array}$ & 4 & 1,18 \\
\hline Melastomataceae & $\begin{array}{l}\text { Tibouchina pulchra } \\
\text { Cogn. }\end{array}$ & manacá-da-serra & nativa & 2 & 0,59 \\
\hline Moraceae & Ficus benjamina L. & $\begin{array}{l}\text { figueira- } \\
\text { benjamina }\end{array}$ & exótica & 5 & 1,47 \\
\hline Moraceae & Ficus insipida Willd. & guaxinguba & nativa & 3 & 0,88 \\
\hline Moraceae & Morus nigra L. & amora-preta & exótica & 1 & 0,29 \\
\hline Morta & Morta & & - & 6 & 1,77 \\
\hline
\end{tabular}




\begin{tabular}{|c|c|c|c|c|c|}
\hline Família & Espécie & Nome popular & Origem & $\mathbf{Q}$ & F\% \\
\hline Myrtaceae & Eucalyptus sp. & & exótica & 2 & 0,59 \\
\hline Myrtaceae & Eugenia involucrata DC. & cereja-do-mato & nativa & 3 & 0,88 \\
\hline Myrtaceae & Eugenia uniflora L. & pitangueira & nativa & 6 & 1,77 \\
\hline Myrtaceae & $\begin{array}{l}\text { Psidium cattleianum } \\
\text { Sabine }\end{array}$ & araçá & nativa & 1 & 0,29 \\
\hline Myrtaceae & Psidium guajava L. & goiabeira & subespontânea & 3 & 0,88 \\
\hline Myrtaceae & $\begin{array}{l}\text { Syzygium cumini (L.) } \\
\text { Skeels }\end{array}$ & jambolão & subespontânea & 40 & 11,80 \\
\hline Myrtaceae & $\begin{array}{l}\text { Syzygium jambos (L.) } \\
\text { Alston }\end{array}$ & jambo & subespontânea & 4 & 1,18 \\
\hline Nyctaginaceae & $\begin{array}{l}\text { Bougainvillea glabra } \\
\text { Choisy }\end{array}$ & primavera & nativa & 1 & 0,29 \\
\hline Nyssaceae & $\begin{array}{l}\text { Camptotheca acuminata } \\
\text { Decne. }\end{array}$ & árvore-feliz & exótica & 11 & 3,24 \\
\hline Oleaceae & $\begin{array}{l}\text { Ligustrum lucidum W.T. } \\
\text { Aiton }\end{array}$ & alfeneiro & subespontânea & 1 & 0,29 \\
\hline Oxalidaceae & Averrhoa carambola L. & carambola & subespontânea & 1 & 0,29 \\
\hline Pittosporaceae & $\begin{array}{l}\text { Pittosporum undulatum } \\
\text { Vent. }\end{array}$ & pau-incenso & subespontânea & 4 & 1,18 \\
\hline Podocarpaceae & $\begin{array}{l}\text { Podocarpus lambertii } \\
\text { Klotzsch ex Endl. }\end{array}$ & pinho-bravo & nativa & 3 & 0,88 \\
\hline Rhamnaceae & Hovenia dulcis Thunb. & mata-fome & subespontânea & 11 & 3,24 \\
\hline Rosaceae & $\begin{array}{l}\text { Eriobotrya japonica } \\
\text { (Thunb.) Lindl. }\end{array}$ & nespera & subespontânea & 5 & 1,47 \\
\hline Rosaceae & Prunus serrulata Lindl. & $\begin{array}{l}\text { cerejeira- } \\
\text { ornamental }\end{array}$ & exótica & 2 & 0,59 \\
\hline Rosaceae & Prunus sp. & & - & 2 & 0,59 \\
\hline Rutaceae & Citrus limon (L.) Osbeck & limoeiro & exótica & 9 & 2,65 \\
\hline Rutaceae & $\begin{array}{l}\text { Murraya paniculata (L.) } \\
\text { Jack }\end{array}$ & murta-de-cheiro & exótica & 2 & 0,59 \\
\hline Sapindaceae & $\begin{array}{l}\text { Allophylus edulis (A.St.- } \\
\text { Hil. et al.) Hieron. ex } \\
\text { Niederl. }\end{array}$ & fruto-de-pombo & $\begin{array}{l}\text { nativa (BR), } \\
\text { exótica (SP) }\end{array}$ & 1 & 0,29 \\
\hline Sapindaceae & $\begin{array}{l}\text { Cupania oblongifolia } \\
\text { Mart. }\end{array}$ & camboatá & nativa & 1 & 0,29 \\
\hline Sapindaceae & $\begin{array}{l}\text { Koelreuteria bipinnata } \\
\text { Franch. }\end{array}$ & árvore-da-china & exótica & 2 & 0,59 \\
\hline Solanaceae & $\begin{array}{l}\text { Brunfelsia uniflora } \\
\text { (Pohl) D. Don }\end{array}$ & $\begin{array}{l}\text { manacá-de- } \\
\text { cheiro }\end{array}$ & nativa & 1 & 0,29 \\
\hline Solanaceae & $\begin{array}{l}\text { Solanum mauritianum } \\
\text { Scop. }\end{array}$ & fumo-bravo & nativa & 1 & 0,29 \\
\hline Urticaceae & Cecropia sp. & & - & 1 & 0,29 \\
\hline Urticaceae & Urera sp. & & - & 1 & 0,29 \\
\hline Verbenaceae & $\begin{array}{l}\text { Citharexylum } \\
\text { myrianthum Cham. }\end{array}$ & pau-viola & nativa & 2 & 0,59 \\
\hline Verbenaceae & Duranta erecta L. & pingo-de-ouro & $\begin{array}{l}\text { nativa (BR), } \\
\text { exótica (SP) }\end{array}$ & 1 & 0,29 \\
\hline
\end{tabular}

A Praça Agostinho Nohama é rica em diversidade de espécies arbóreas, assim como cinco praças da região central do município de Cachoeira do Sul (RS) e, a Praça Sete de Setembro em Ribeirão Preto, interior de São Paulo (REDIN et al., 2010;
GIMENES et al., 2011). As famílias mais representativas em ambos os trabalhos, coincidem com as deste estudo, sendo elas, Fabaceae (14), Myrtaceae (7), Arecaceae (6) e Bignoniaceae (6). 
As espécies mais frequentes são Syzygium cumini, com 40 indivíduos, Syagrus romanzoffiana (23), Spathodea nilotica (19), Cupressus sempervirens (16), e Terminalia catappa (15), destas apenas $S$. romanzoffiana é nativa.

A família Fabaceae $(38,9 \%)$ e a espécie $S$. cumini $(11,8 \%)$ estão acima dos índices recomendados por Santamour Junior (1990), tal situação pode afetar a sanidade das árvores deste local.

No estado de Minas Gerais, em Uberlândia, Rezende \& Santos (2010) verificaram uma situação diferenciada dos trabalhos citados. Os autores estudaram cinco praças, e verificaram que $72,9 \%$ da arborização são compostas por apenas cinco espécies, além disso, afirmam que a prática de cultivar muitos indivíduos sem variabilidade de espécies é algo comum em outros municípios, como Maringá (PR), Piracicaba (SP) e Porto Alegre (RS). Outro aspecto relevante é a utilização de espécies exóticas na arborização urbana. Neste estudo, elas representam 37,7\% (SP) e 30,6\% (BR) do total amostrado (Figura 1.). Apesar do índice elevado, outras regiões apresentam situações mais críticas, como em Lavras da Mangabeira (CE), onde 92,95\% da vegetação da cidade são exóticas ou em Uberlândia (MG), onde o percentual ultrapassa a ordem dos 60 pontos (CALIXTO JÚNIOR et al., 2009; REZENDE e SANTOS, 2010).

Segundo Blum et al. (2008), as exóticas invasoras podem afetar diretamente a biodiversidade, a economia e a saúde humana, e ocupam o segundo lugar na lista das causas de extinções das espécies no mundo. No caso de Maringá (PR), os autores concluíram que apenas $24,1 \%$ da vegetação urbana são nativas, já para a Praça Agostinho Nohama, o percentual corresponde a 32,9. Recomenda-se utilizar espécies nativas regionais para possível estabelecimento de corredores ecológicos.

Figura 1. Origens das espécies registradas na Praça Agostinho Nohama, considerando-se tanto o Brasil, como o estado de São Paulo

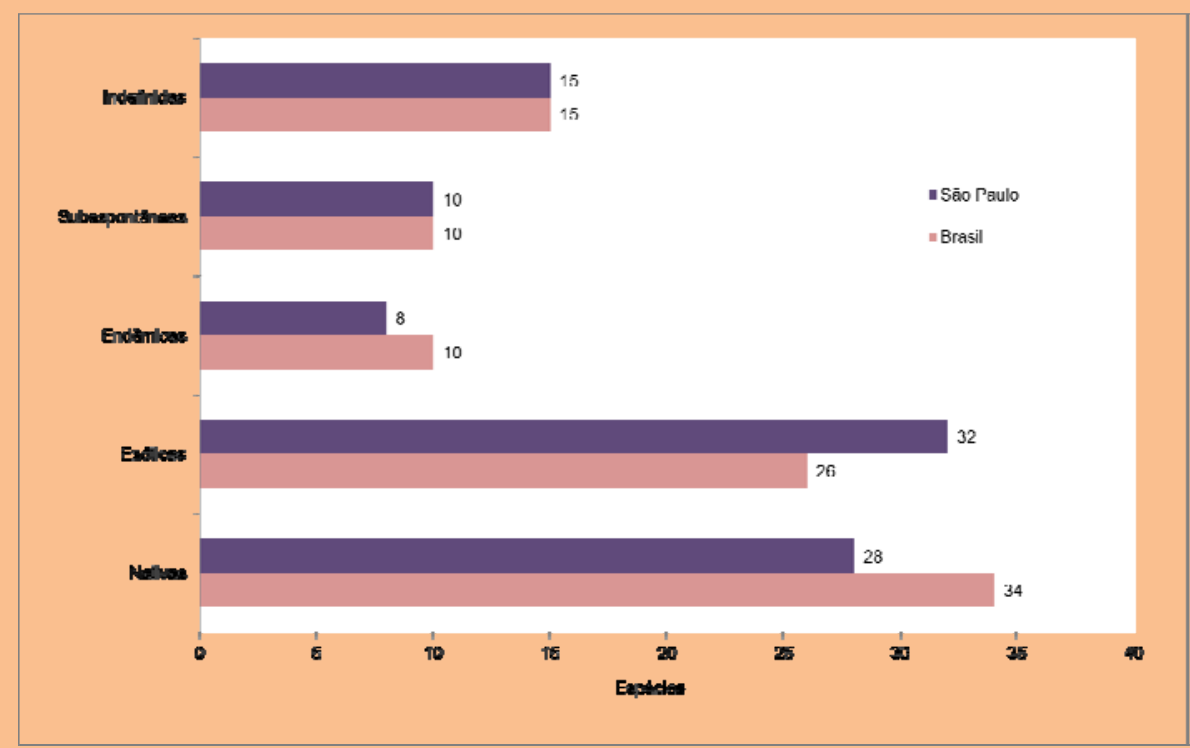


As alturas e as circunferências das árvores foram classificadas (Tabela 2). A altura média das árvores é de $11,7 \mathrm{~m}$ e a distribuição das alturas (Figura 2) mostra que apenas 66 indivíduos (19,5\%) têm altura menor que $5 \mathrm{~m}$, e mais de $80 \%$ apresentam altura maior que 10 metros, além disso, $53 \%$ dos espécimes têm o CAP acima de $50 \mathrm{~cm}$, esses resultados evidenciam que a maioria das amostras é adulta.

Tabela 2. Classes de altura e circunferência a altura do peito (CAP)

\begin{tabular}{ccc}
\hline Classes & Altura $(\mathbf{m})$ & CAP $\mathbf{( c m})$ \\
\hline 1 & $1-5$ & $10-50$ \\
2 & $>5-10$ & $>50-100$ \\
3 & $>10-15$ & $>100-150$ \\
4 & $>15-20$ & $>150-200$ \\
\hline 5 & $>20$ & $>200$ \\
\hline
\end{tabular}

Figura 2. Distribuição em classes de altura e circunferência das árvores quantificadas na Praça Agostinho Nohama

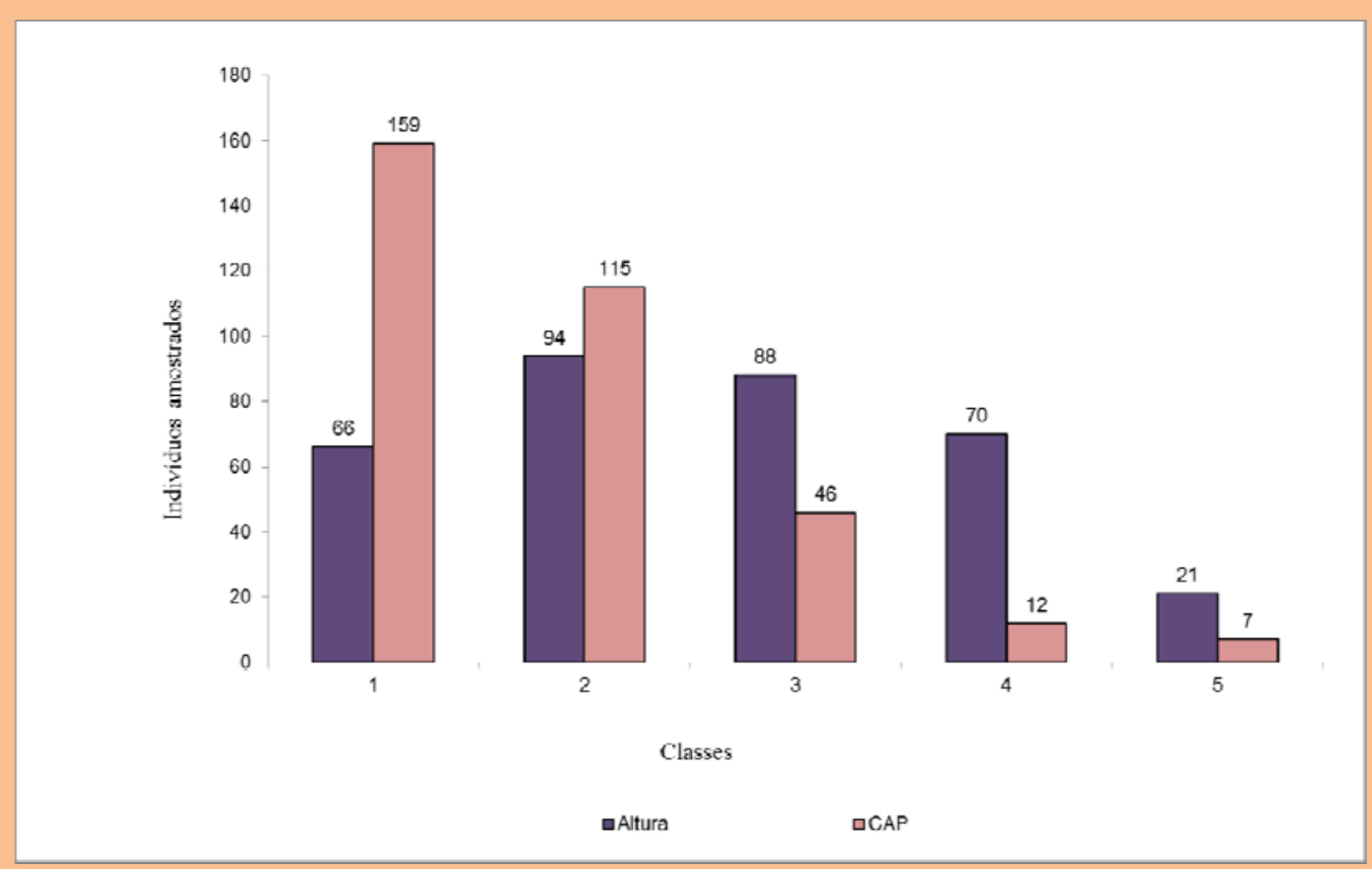

Em relação às árvores com CAP $>200 \mathrm{~cm}$, a Ceiba speciosa (paineira) foi a mais representativa, com três indivíduos, seguida por Eucalyptus sp. (dois

\section{Qualitativos}

As condições gerais dos espécimes amostrados podem ser visualizadas na figura 3 , onde $85,3 \%$ estão em bom estado, $11,5 \%$ necessitam de espécimes), Jacaranda mimosifolia (um) e Ficus benjamina (um). 
risco aos frequentadores da praça. As espécies que estão em estado ruim são: Casuarina sp., Enterolobium contortisiliquum (tamboril) (Figura 4), Citharexylum myrianthum (pau-viola), Piptadenia gonoacantha (pau-jacaré), e Eucalyptus sp. A maior porcentagem de espécimes em estado bom coube ao Syzygium cumini, com 11,8\%, seguido do Syagrus romanzoffiana (8\%).

Foi observado que a predação natural (Figura 5) por insetos, fungos e/ou doenças, ocorreram em 38\% podendo variar a intensidade de leve a grave, e o vandalismo (Figura 6) ocasionado por seres humanos, foi notado em $19,2 \%$ das árvores amostradas.
No caso de Águas de São Pedro (SP), Bortoleto (2004) afirma que o vandalismo é um ato raro no local, o que difere dos resultados do presente estudo, pois apesar da maioria dos indivíduos estarem em bom estado, tais injúrias mecânicas é comum na área.

Para Milano (1987) apud Rezende e Santos (2010) o vandalismo é o resultado do desinteresse da comunidade em participar de programas de arborização e ainda a falta de consciência sobre a importância dos espécimes vegetais.

Figura 3. Estado geral dos espécimes amostrados na Praça Agostinho Nohama

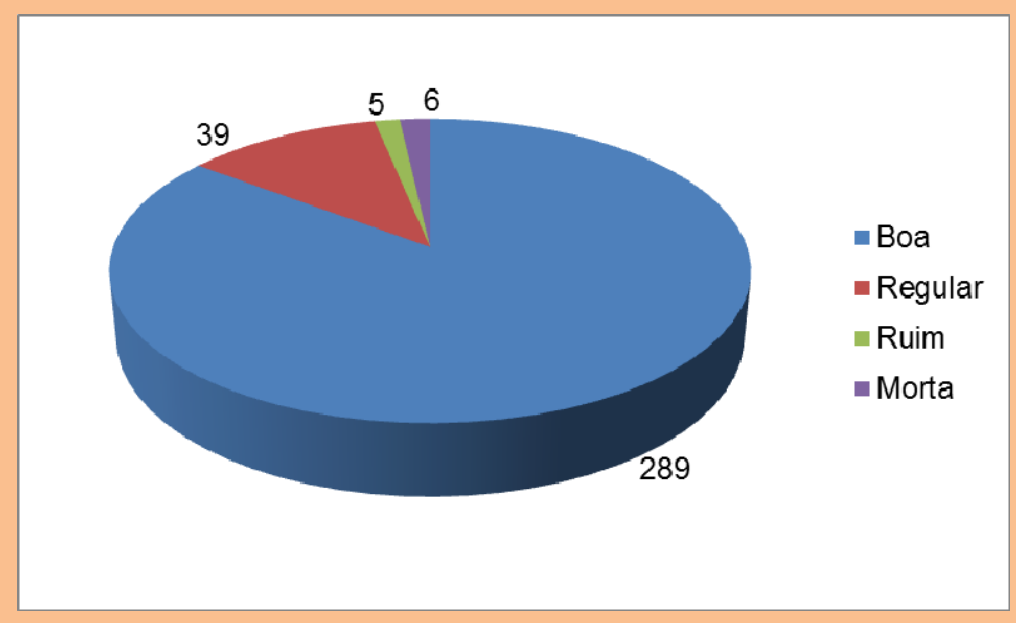

Figura 4. Enterolobium contortisiliquum comprometido pelo seu estado de conservação

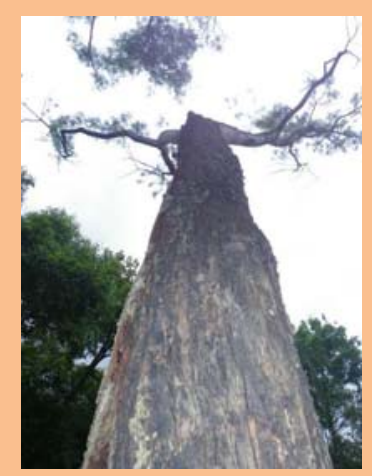

Fonte: Lucas Teixeira Alves da Silva 24/09/2011

AVALIAÇÃO QUALI-QUANTITATIVA DA ARBORIZAÇÃO... 
Figura 5. Lesão grave, ocasionada por insetos

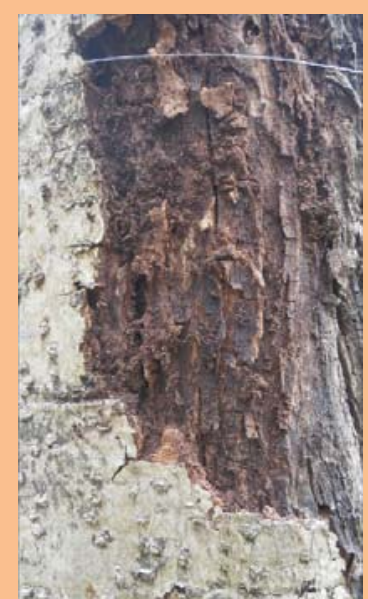

Fonte: Lucas Teixeira Alves da Silva 24/09/2011

Figura 6. Inscrições no caule, forma de vandalismo observado na Praça

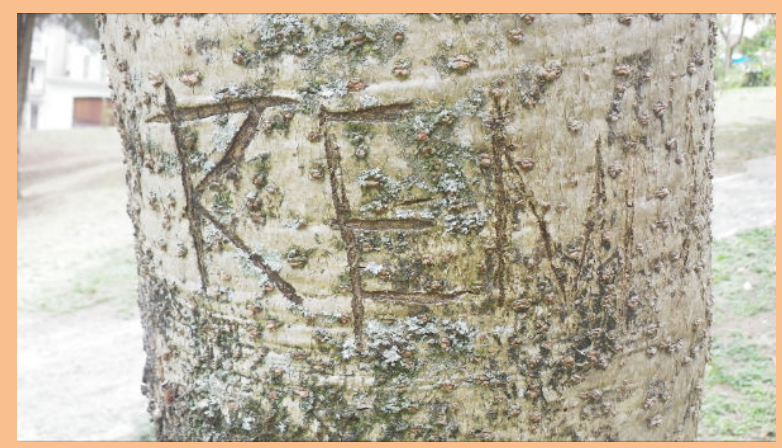

Fonte: Lucas Teixeira Alves da Silva 24/09/2011

Embora exista predação por seres humanos, outros organismos relacionam-se com as árvores de maneira diferenciada, neste caso, a Praça apresenta papel importante na associação ecológica, 282 $(83,2 \%)$ indivíduos possuem interações com líquens, 264 (77,9\%) com insetos, e 169 (49,8\%) com epífitas, a figura 7, ilustra essa distribuição.

Os insetos e as aves auxiliam na polinização e na dispersão de sementes, propiciando maior fluxo gênico e controle populacional das espécies vegetais (FONSECA et al., 2008; LUCCAS et al., 2009). Na Praça foram observados pássaros em 30 árvores, utilizando-as como poleiros ou alimentando-se dos frutos e/ou sementes, corroborando as conclusões de Brun et al. (2007) e Shams et al. (2009), onde afirmam que a utilização de árvores em áreas urbanas auxilia na manutenção ecológica e favorecem a avifauna.

Observaram-se aves da Família Psittacidae alimentando-se de sementes de Spathodea nilotica, essa interação é benéfica, pois reduz a disponibilidade de sementes, e consequentemente, controla a proliferação destas árvores exóticas.

As fases reprodutivas dos espécimes vegetais podem ser observadas na tabela 3 , podendo ser representadas por flor e/ou fruto (estróbilo para as 
Figura 7. Distribuição ecológica das formas de vida nas árvores amostradas

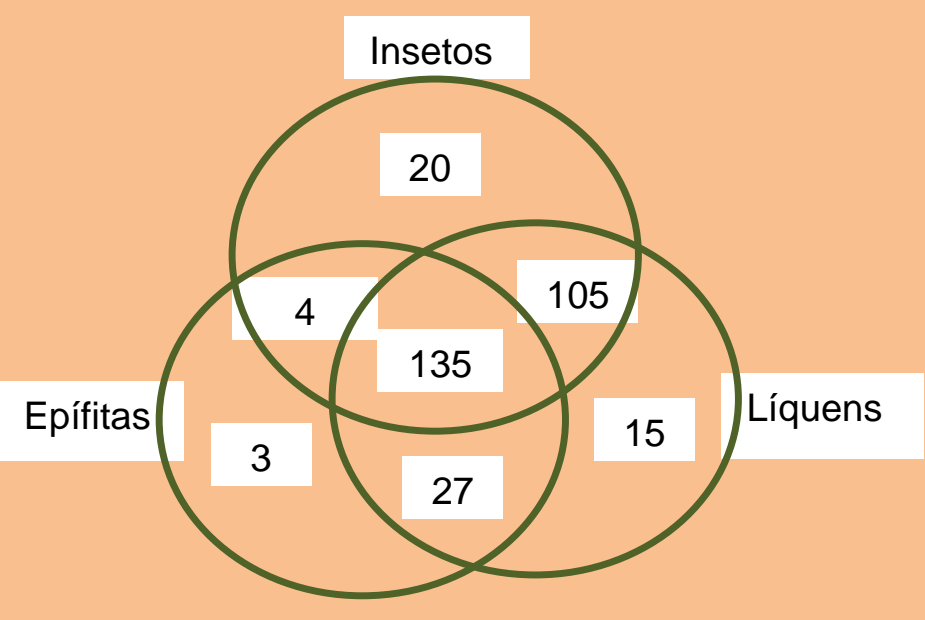

Tabela 3. Espécies, fase reprodutiva (Flor, Fruto) e Frequência Absoluta (Q). As observações ocorreram no mês de setembro de 2011

\begin{tabular}{|c|c|c|c|}
\hline Espécie & Flor & Fruto & $\mathbf{Q}$ \\
\hline Averrhoa carambola & $\mathrm{X}$ & & 1 \\
\hline Archontophoenix cunninghamii & & $\mathrm{X}$ & 3 \\
\hline Bougainvillea glabra & $\mathrm{X}$ & & 1 \\
\hline Brunfelsia uniflora & $\mathrm{X}$ & & 1 \\
\hline Camptotheca acuminata & $\mathrm{X}$ & $\mathrm{X}$ & 10 \\
\hline Citrus limon & $\mathrm{X}$ & $\mathrm{X}$ & 5 \\
\hline Cupania oblongifolia & & $\mathrm{X}$ & 1 \\
\hline Cupressus sempervirens & & $\mathrm{X}$ & 11 \\
\hline Cupressus sp. & & $\mathrm{X}$ & 1 \\
\hline Delonix regia & & $\mathrm{X}$ & 1 \\
\hline Dendropanax cuneatus & & $\mathrm{X}$ & 1 \\
\hline Duranta erecta & $\mathrm{X}$ & & 1 \\
\hline Dypsis lutescens & $\mathrm{X}$ & & 1 \\
\hline Enterolobium contortisiliquum & & $\mathrm{X}$ & 1 \\
\hline Eriobotrya japonica & & $\mathrm{X}$ & 4 \\
\hline Erythrina speciosa & $\mathrm{X}$ & & 1 \\
\hline Eugenia uniflora & $\mathrm{X}$ & $\mathrm{X}$ & 6 \\
\hline Ficus benjamina & & $\mathrm{X}$ & 1 \\
\hline Hovenia dulcis & & $\mathrm{X}$ & 2 \\
\hline Jacaranda mimosifolia & & $\mathrm{X}$ & 1 \\
\hline Lafoensia pacari & $\mathrm{X}$ & & 2 \\
\hline Livistona chinensis & & $\mathrm{X}$ & 1 \\
\hline Malvaviscus arboreus & $\mathrm{X}$ & & 2 \\
\hline Mangifera indica & $\mathrm{X}$ & & 2 \\
\hline Morus nigra & & $\mathrm{X}$ & 1 \\
\hline Murraya paniculata & & $\mathrm{X}$ & 1 \\
\hline Nerium oleander & $X$ & & 1 \\
\hline Ocotea pichurim & & $\mathrm{X}$ & 1 \\
\hline
\end{tabular}




\begin{tabular}{|c|c|c|c|}
\hline Espécie & Flor & Fruto & $\mathbf{Q}$ \\
\hline Persea americana & $\mathrm{X}$ & $\mathrm{X}$ & 3 \\
\hline Piptadenia gonoacantha & & $\mathrm{X}$ & 4 \\
\hline Pittosporum undulatum & $\mathrm{X}$ & $\mathrm{X}$ & 3 \\
\hline Prunus serrulata & & $\mathrm{X}$ & 2 \\
\hline Prunus sp. & & $\mathrm{X}$ & 2 \\
\hline Pseudobombax sp. & & $\mathrm{X}$ & 1 \\
\hline Schefflera arboricola & $\mathrm{X}$ & & 1 \\
\hline Schinus molle & $\mathrm{X}$ & & 1 \\
\hline Spathodea nilotica & & $\mathrm{X}$ & 13 \\
\hline Syagrus romanzoffiana & $\mathrm{X}$ & $\mathrm{X}$ & 2 \\
\hline Syzygium cumini & $\mathrm{X}$ & $\mathrm{X}$ & 3 \\
\hline Syzygium jambos & $\mathrm{X}$ & $\mathrm{X}$ & 3 \\
\hline Terminalia catappa & $\mathrm{X}$ & $\mathrm{X}$ & 15 \\
\hline Tibouchina granulosa & & $\mathrm{X}$ & 2 \\
\hline Vernonanthura divaricata & & $\mathrm{X}$ & 2 \\
\hline
\end{tabular}

Segundo dados meteorológicos do Instituto Florestal (2011), foram quantificados apenas $2 \mathrm{~mm}$ de precipitação no mês de setembro, mesmo com pouca água disponível no ambiente, os indivíduos férteis representam $36,6 \%$ do total amostrado.

A Praça apresenta importante papel ecológico, pelas diversas interações já descritas, e também, por estar próxima do Parque Estadual da Cantareira (PEC), onde Arzolla (2002) no núcleo Águas Claras descreve 194 espécies, destas, seis encontram-se na lista de espécies ameaçadas de extinção, sendo elas, Beilschmiedia emarginata, Euplassa cantareirae,
Ocotea catharinensis, Ocotea odorifera, Roupala brasiliensis e Trichilia silvatica.

Com o intuito de auxiliar o estabelecimento destas espécies e contribuir com a diversidade e manutenção biológica, sugerem-se o plantio das espécies apresentadas no trabalho de Arzolla (2002). Sendo assim, a Praça pode ser utilizada como ferramenta para projetos de educação ambiental.

\section{CONCLUSÃO}

A Praça Agostinho Nohama é rica em diversidade de espécies arbóreas, porém o índice de espécies exóticas é elevado. No geral, apresenta bom estado de conservação, todavia foram observadas injúrias físicas e biológicas em alguns espécimes, para tanto, recomenda-se a supressão de onze indivíduos que estão com lesões graves, oferecendo risco aos frequentadores, e aos exemplares sadios.
Infere-se que a Praça tem potencial para atuar como corredor ecológico, por sua proximidade com o Parque Estadual da Cantareira, sendo assim, sugerese o plantio das espécies nativas deste complexo, a fim de contribuir com a diversidade regional e possibilitar trabalhos de educação ambiental. 
Aos moradores do bairro, em especial ao Senhor Aparecido Cabreira, pela dedicação a conservação da Praça. Aos pesquisadores do Instituto Florestal do Estado de São Paulo, Daniela Fessel Bertani, João Aurélio Pastore, pelo apoio as identificações e as sugestões. Á Rochelle Lima Ramos dos Santos, e a Maria Aparecida da Costa Sousa, por auxiliarem nos trabalhos de campo. Á Juliana Maerschner A. Peixoto, pelas correções.

\section{REFERÊNCIAS BIBLIOGRÁFICAS}

ABREU, L. V. Avaliação da escala de influência da vegetação no microclima por diferentes espécies arbóreas. Campinas, 2008. 154 p. Dissertação (Mestrado em Engenharia Civil) - Universidade Estadual de Campinas, 2008.

ABREU, L.V.; LABAKI, L.C. Conforto térmico proporcionado por algumas espécies arbóreas: avaliação do raio de influência através de diferentes índices de conforto. Ambiente Construído, Porto Alegre, v. 10, n. 4, p. 103117,2010 .

AMARAL, R.D.A.M.; BRAZOLIM, S.; LIMA, A.M.L.P.; BARILLARI, C.T. Ocorrência de organismos xilófagos em árvores de pau-brasil (compact disc). In: CONGRESSO BRASILEIRO DE ARBORIZAÇÃO URBANA, 7., Belém, 2003. Resumos. Belém: UFPA, 2003.

ARZOLLA, F.A.R.D.P. Florística e fitossociologia de trecho da Serra da Cantareira, núcleo Águas Claras, Parque Estadual da Cantareira, Mairiporã - SP. Campinas, 2002. 184 p. Dissertação (Mestrado em Biologia Vegetal) - Universidade Estadual de Campinas, 2002.

BLUM, C. T.; BORGO, M.; SAMPAIO, A. C. F. Espécies exóticas invasoras na arborização de vias públicas de Maringá - PR. Rev. SBAU, Piracicaba, v. 3, n. 2, p. 78-97, 2008.

BORTOLETO, S. Inventário quali-quantitativo da arborização viária da estância da Águas de São Pedro SP. Piracicaba, 2004. 85p. Dissertação (Mestrado em Agronomia) - Escola Superior de Agricultura "Luiz de Queiroz", Universidade de São Paulo, 2004.

BRUN, F. G. K.; LINK, D.; BRUN, E. J. O emprego da arborização na manutenção da biodiversidade de fauna em áreas urbanas. Rev. SBAU, Piracicaba, v. 2, n. 1, p. 117-127, 2007.

CALIXTO JÚNIOR, J. T.; SANTANA, G. M.; LIRA FILHO, J. A. Análise quantitativa da arborização urbana de Lavras da Mangabeira, CE, Nordeste do Brasil. Rev. SBAU, Piracicaba, v. 4, n. 3, p. 99-109, 2009.

FILHO, A. P. A.; RIBEIRO, H. A percepção do caos urbano, as enchentes e suas repercussões nas políticas públicas da Região Metropolitana de São Paulo. Saúde e Sociedade, São Paulo, v. 15, n. 3, p. 145-161, 2006. 
FONSECA, L.C.N.; ALMEIDA, E.M.; ALVES, M.A.S. Fenologia, morfologia floral e visitantes de Psychotria brachypoda (Müll. Arg.) Britton (Rubiaceae) em uma área de Floresta Atlântica, Sudeste do Brasil. Rev. Acta bot. bras., São Paulo, v. 22, n. 1, p. 63-69, 2008.

FORZZA, R.C.; LEITMAN, P.M.; COSTA, A.F.; CARVALHO JR., A.A.; PEIXOTO, A.L.; WALTER, B.M.T.; BICUDO, C.; ZAPPI, D.; COSTA, D.P.; LlERAS, E.; MARTINELlI, G.; LIMA, H.C.; PRADO, J.; STEHMANN, J.R.; BAUMGRATZ, J.F.A.; PIRANI, J.R.; SYLVESTRE, L.; MAIA, L.C.; LOHMANN, L.G.; QUEIROZ, L.P.; SILVEIRA, M.; COELHO, M.N.; MAMEDE, M.C.; BASTOS, M.N.C.; MORIM, M.P.; BARBOSA, M.R.; MENEZES, M.; HOPKINS, M.; SECCO, R.; CAVALCANTI, T.B.; SOUZA, V.C. Introdução. In: Lista de Espécies da Flora do Brasil. Jardim Botânico do Rio de Janeiro. 2010.

GIMENES, R.; ROMANI, G. N.; BATISTAL, G. S.; PIVETTA, K. F. L. Interpretação do uso, do imobiliário e da arborização da Praça Sete de Setembro, Ribeirão Preto, SP. Rev. SBAU, Piracicaba, v. 6, n. 3, p. 22-42, 2011.

INSTITUTO FLORESTAL DO ESTADO DE SÃO PAULO. Dados Meteorológicos. Disponível em: <http://www.iflorestal.sp.gov.br/servicos/index.asphttp://www.tropicos.org/>. Acesso em: 30 out. 2011

LUCCAS, N.I.; HALLER, E.C.P.; ANTUNES, A.Z. Dieta de Psitacídeos (Aves: Psittacidae) no Parque Estadual Aberto Löfgren - SP. IF Sér. Reg., São Paulo, n. 40, p. 143-147, 2009.

MILANO, M.S.; NUNES, M.L.; SANTOS L.A dos; SARNOWSKI FILHO, O.; ROBAYO, J.A.M. Aspectos qualiquantitativos da arborização de ruas de Curitiba (1991). In: CONGRESSO BRASILEIRO SOBRE ARBORIZAÇÃO URBANA, 1.; ENCONTRO NACIONAL SOBRE ARBORIZAÇÃO URBANA, 4., Vitória, 1992. Anais. Vitória: Prefeitura, v.2, p.199-210, 1992.

MOBOT - Missouri Botanical Garden. 2008. Disponível em: $<$ http://www.tropicos.org/>. Acesso em: 01 out. 2011.

MOREIRA, T. C. L. Interação da vegetação arbórea e poluição atmosférica na cidade de São Paulo. Piracicaba, 2010. 79 p. Dissertação (Mestrado em Recursos Florestais) - Escola Superior de Agricultura "Luiz de Queiroz", Universidade de São Paulo, 2010.

MUNEROLI, C. C.; MASCARÓ, J. J. Arborização urbana: uso de espécies arbóreas nativas na captura do carbono atmosférico. Rev. SBAU, Piracicaba, v. 5, n. 1, p. 160-182, 2010.

PAIVA, G. A. Levantamento florístico quali-quantitativo da Praça do Relógio da Cidade Universitária “Armando de Salles Oliveira” - USP/São Paulo. Piracicaba, 2006. 116 p. Dissertação (Mestrado em Agronomia) - Escola Superior de Agricultura “Luiz de Queiroz”, Universidade de São Paulo, 2006.

REDIN, C. G.; VOGEL, C.; TROJAHN, C. D. P.; GRACIOLI, C. R.; LONGHI, S. J. Análise da arborização urbana em cinco praças do município de Cachoeira do Sul, RS. Rev. SBAU, Piracicaba, v. 5, n. 3, p. 149-164, 2010.

REZENDE, T. M.; SANTOS, D. G. Avaliação quali-quantitativa da arborização das praças do bairro Jaraguá, Uberlândia, MG. Rev. SBAU, Piracicaba, v. 5, n. 2, p. 139-157, 2010.

ROCHA, R. T.; LELES, P. S. S.; OLIVEIRA NETO, S. N. Arborização de vias públicas em Nova Iguaçu, RJ: o caso dos bairros Rancho Novo e Centro. R. Árvore, Viçosa-MG, v. 28, n. 4, p. 599-607, 2004.

SANCHOTENE, M.C.C. Desenvolvimento e perspectivas da arborização urbana no Brasil. In: Congresso Brasileiro de Arborização Urbana, 2, 1994. São Luís - MA. Anais. Sociedade Brasileira de Arborização Urbana, 1994.

SANTAMOUR JUNIOR, F.S. Trees for urban planting: diversity, uniformity, and common sense. In: METRIA CONFERENCE, 7., 1990, Lisle. Proceedings... Lisle: 1990. p.57-66.

SEADE - FUNDAÇÃO SISTEMA ESTADUAL DE DADOS. Censo 2010. Disponível em: $<$ http://www.seade.gov.br/index.php?option=com_content\&view=article\&id=1070\&Itemid=54> Acesso em:05 out. 2011.

SHAMS, J. C.; GIACOMELI, D. C.; SUCOMINE, N. M. Emprego da arborização na melhoria do conforto térmico nos espaços livres públicos. Rev. SBAU, Piracicaba, v. 4, n. 4, p. 1-16, 2009.

SILVA, L. F. Interceptação da chuva nas espécies de sibipiruna (Caesalpinia pluviosa DC.) e tipuana (Tipuana tipu O. Kuntze). Piracicaba, 2008. 60 p. Tese (Doutorado em Agronomia) - Escola Superior de Agricultura "Luiz de Queiroz", Universidade de São Paulo, 2008.

SOUZA, M.A.L.B.; BUENO, O.C. Planejamento da arborização urbana. In: CONGRESSO BRASILEIRO DE ARBORIZAÇÃO URBANA, 5.; Anais. Rio de Janeiro, 2000.

Rita de Cássia Sousa et al. 
SOUZA, V. C.; LORENZI, H. Botânica Sistemática: guia ilustrado para identificação das famílias de Angiospermas da flora brasileira, baseado em APG II. 2 ed. Instituto Plantarum, Nova Odessa, 2007.

TAKAHASHI, L.Y. Arborização urbana: inventário. In: CONGRESSO BRASILEIRO DE ARBORIZAÇÃO URBANA, 2.; ENCONTRO NACIONAL SOBRE ARBORIZAÇÃO URBANA, 5., São Luís, 1994. Anais. São Luís: Sociedade Brasileira de Arborização Urbana, p. 193-199, 1994.

TAKIYA, H. Atlas ambiental do Município de São Paulo - Fase I: diagnóstico e bases para a definição de políticas públicas para as áreas verdes no município de São Paulo. Fapesp, Prefeitura do Município de São Paulo, Secretaria Municipal do Meio Ambiente e Secretaria Municipal de Planejamento Urbano, São Paulo - SP, 2002.

TARIFA, J. R.; ARMANI, G. Atlas Ambiental do Município de São Paulo - Fase I: diagnóstico e bases para a definição de políticas públicas para as áreas verdes no município de São Paulo. Unidades Climáticas Urbanas da Cidade de São Paulo. Laboratório de Climatologia. Departamento de Geografia/ Faculdade de Filosofia, letras e Ciências Humanas - Universidade de São Paulo, 2000.

ZILLER, S.R. Plantas exóticas invasoras: a ameaça da contaminação biológica. Cienc. Hoje, v. 30, n. 178, p. 77-79, 2001. 